\title{
Short-Term Outcomes in Elderly Patients with SARS-CoV-2 Infections Discharged from Community Hospitals
}

\author{
Spence D, Dexter J, Barclay C, Latham S, Richardson A and Ghosh S*
}

Community Health Services, Leicestershire Partnership Trust, ${ }^{*}$ Corresponding author

Leicester School Allied Health Sciences, DMU

Ghosh S, Faculty of Health Sciences, De-Montfort University, Leicester UK, Enderby Medical Centre, Leicestershire, UK

Submitted:23 March 2021; Accepted: 01 Apr 2021; Published: 07 Apr 2021

Citation: Dexter J, Barclay C, Latham S, Richardson A, Ghosh S, et al. (2021) Short-term Outcomes in elderly patients with SARSCoV-2 infections discharged from Community Hospitals. Journal of Clinical Review \& Case Reports 6(4): 609-611.

\begin{abstract}
Introduction: Clinical outcomes from very elderly hospitalised patients with SARS-COV-2 (Covid-19), are poor, with a reported in-patient mortality as high as 20\%. Age, sex, comorbidities, ethnicity and deprivation have all been shown to correlate with worse outcomes in patients with Covid-19, however the outcomes of elderly hospitalised patients once discharged remains unknown. We looked at our cohort of very elderly patients treated within community hospital settings.
\end{abstract}

Methods \& Results: A retrospective case note analysis was undertaken within our 222 bedded community hospital structure during the first wave of the pandemic from April to August 2020. A total of 129 patients were treated during this period. The average age of the patients was $78.8 \pm 2.7$ years. In patient mortality was $11 \%$ (14 patients). $83 \%$ of patients were discharged from the hospitals. $27 \%$ were discharged to care home facilities. At 90 days, $83 \%$ of patients were still alive but a third of these patients were re-admitted into hospital with various different diagnoses.

Conclusion: Elderly patients who survived Covid-19 infection and left hospital have a relatively high mortality rate in the short-term. Also connected with this is a high morbidity rate and therefore care must be directed within primary care to ensure that this group stay out of hospitals.

\section{Introduction}

Leicestershire Partnership NHS Trust (LPT) is a university teaching trust in the East Midlands. Leicester, Leicestershire and Rutland (LLR) has a $49 / 51 \%$ ethnic diversity split with a population about 1.2 million people [1]. Community Services provision is delivered through Leicestershire Partnership Trust, non-acute trust within LLR. Within the division, there are 8 community hospitals with medical cover delivered by Consultant Geriatrician support of 2 sessions a week and day to day medical support delivered using advanced nurse practitioners. The sites spread over the wide geographical area of Leicester, Leicestershire and Rutland. There are currently 222 community hospital beds and within this are 30 stroke rehabilitation beds.

During the initial Covid-19 pandemic, LPT community hospitals responded by creating a 'red' ward dedicated to the care and rehabilitation of Covid-19 pneumonitis patients whilst continuing to admit patients for rehabilitation and medical step down from our acute trust partners to other wards. The main referrer was University Hospitals of Leicester (UHL). LPT's Covid-19 ward was created at a site that did not house any other services to reduce contact and risk to non Covid-19 patients/carers.

Clinical outcomes from hospitalised patients with Covid-19 are poor, with a reported mortality of $21 \%[2,3]$. Age, sex, comorbidities, ethnicity and deprivation have all been shown to correlate with worse outcomes in patients with Covid-19, however the outcomes of hospitalised patients once discharged remains unknown [4-6].

We have reviewed data from those positive patients within the community hospitals including those admitted directly to the red 
ward, looking specifically at hospital outcome and at 30/60/90 days' post discharge. The aim of the work was to identify the number of patients readmitted to hospital after 30/60/90 days.

\section{Methods}

Retrospective data analysis was performed between March 2020, when the first confirmed patient was identified within LPT, and August 2020. During this period a total of 129 patients were cared for within the LPT hospitals with a Covid-19 diagnosis. Their presentation was Covid pneumonitis to the acute sector before being transferred to the LPT setting for on-going care.

\section{Results}

129 Covid-19 patients were admitted to LPT during this period. The hospital outcomes are displayed in chart 1. Average age of the patients were $78.8 \pm 2.7$ years and female: male ratio was 1.4:1. 14 patients died prior to discharge, 9 patients required readmission back to secondary acute care (UHL) and 106 patients were discharged alive. 34 patients were discharged to a residential care home facility. The average length of stay for this cohort of patients who were discharged was $15.7 \pm 4.3$ days.

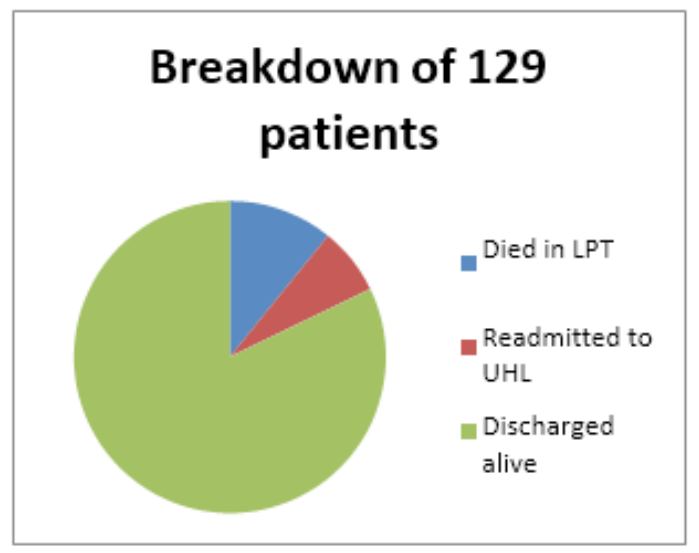

Chart 1: Hospital outcomes of patients with Covid-19 diagnosis

\section{Outcome Review}

Following discharge of the 106 patients discharged from our community hospitals, 18 patients died within the 90 days. The breakdown of the mortality figures is shown in Table 1 below.

\begin{tabular}{|c|c|}
\hline Days post discharge & Mortality (n) \\
\hline$<28$ days & 11 \\
\hline 29-60 days' post discharge & 6 \\
\hline $61-90$ days' post discharge & 1 \\
\hline
\end{tabular}

Of the patients that died following a diagnosis of Covid-19, 14 of the 18 patients were re-admitted to the acute sector and died in hospital.

\section{Readmission data}

Following discharge from community hospital in-patient wards, 36 patients re-presented to secondary care via the emergency department. The data is presented in Table 2 below.

\begin{tabular}{|c|c|}
\hline Days post discharge & Readmission $(\mathrm{n})$ \\
\hline$<28$ days & 22 \\
\hline 29-60 days post discharge & 11 \\
\hline 61-90 days post discharge & 3 \\
\hline
\end{tabular}

These patients re-presented with a number of elements, all which can be related to Covid-19 and increased frailty of old age. The data is highlighted in Table 3

\section{Table 3: Readmission diagnoses for Covid 19 discharges}

\begin{tabular}{|c|c|}
\hline Diagnosis & No. of patients (\%) \\
\hline Fall & $20(56 \%)$ \\
\hline Leg swelling/ Leg pain & $4(12 \%)$ \\
\hline Acute Kidney Injury & $2(6 \%)$ \\
\hline Shortness of breath & $4(12 \%)$ \\
\hline Abdominal pain & $2(6 \%)$ \\
\hline Generalised weakness & $1(3 \%)$ \\
\hline New onset Atrial Fibrillation & $2(6 \%)$ \\
\hline Chest Pain & $1(3 \%)$ \\
\hline Pneumonia & $2(6 \%)$ \\
\hline
\end{tabular}

In comparison with LPTs readmission rates for our community hospitals at 30 days, Covid-19 patients had a slightly higher rate of $23 \%$, compared to $18.8 \%$ in $2019-20$.

\section{Discussion}

During the first wave of the pandemic, the initial Covid-19 infection control response during the first inpatient outbreaks was to transfer PCR-positive patients across to our community hospitals for medical step down care and following rehabilitation for this elderly group of patients. Patients admitted to our rehabilitation wards were PCR positive on admission and underwent regular surveillance Covid-19 testing. When they were found to be Covid-19 negative, the patient was transferred to a non-Covid-19 rehabilitation ward to carry on rehabilitation and discharge planning. While there were varying policies in test of cures during three months of the outbreak, $\mathrm{x} 2$ negative swabs was mandatory for all care home discharges due to serious clinical risk posed to the existing residents. This study showed that in our first cohort of 129 patients, the majority of the cohort were discharged from hospital and in patient mortality was $11 \%$. It is clear from current literature that Covid-19 disproportionally affects older patients due to diminished immune function and multi-morbidities [7]. This patient group is most likely to require hospital admission, and most likely to die from Covid-19 infection. Significant physical functional decline is a recognised outcome in the surviving geriatric Covid-19 population and our study demonstrated this though quantifiable deterioration in mobility, increase in care status and increased frailty post-Covid infection. During the wave, our staff worked closely with social services and implemented a discharge hub to streamline admissions and discharges. The discharge process was simplified with the addition of extra Covid-19 funding and the reduction of delay of social care funding checklists and 
funding meetings. This probably accounted for the reduction we demonstrated in the average LOS for Covid-19 patients being 15.7 days compared to LOS of 17 days for our non Covid patients during 2019-20.

This is the first study that reports on the clinical outcomes of this very elderly cohort in the short term following discharge from hospital. Within 3 months, a further 14\% mortality was observed in this group. A significant proportion (28\%) also were re-admitted to acute health facilities with multiple morbidities. The largest cause of readmission from home was falls, accounting for $47 \%$ of the readmissions. This is broadly in line with a report recently published by Ayoubkhani et al [8]. In a more recent study of 1,775 US veterans hospitalised with COVID-19, 20\% were readmitted and $9 \%$ died within 60 days of discharge [9]. With over 4 million people in the UK having tested positive for COVID-19 at the time of writing, and many more who had the disease but never received a test, our findings suggest that the long term burden of COVID-19 related morbidity on hospitals and broader healthcare systems is likely to be substantial in this elderly group [10]. Vaccinations hopefully will protect this susceptible vulnerable but vaccine coverage will not be entirely $100 \%$ protective. There will still be a substantial burden to our healthcare system.

This study has several limitations. It is a single-center retrospective study with a limited patient number, and we considered only the outcome data but not included the risk

stratification data and treatment modalities. However, it was the aim of this study to describe the clinical outcomes of very elderly Covid-19 patients discharged from hospital. Secondly, like all observational studies, we cannot rule out the possibility of residual confounding variables which precludes our ability to draw definitive causal conclusions. Thirdly, a retrospective look at frailty scores and outcomes would be a further area for investigation as emphasis on frailty scores is increasing.

In conclusion, elderly patients who survived Covid-19 infection and left hospital have a relatively high mortality rate in the short-term. Also connected with this is a high morbidity rate and therefore care must be directed within primary care to ensure that this group stay out of hospitals.

\section{References}

1. https://www.ukpopulation.org/leicester-population,

2. Report of the WHO-China Joint Mission on Coronavirus Disease 2019 (COVID-19) (2020) https://www.who.int/ publications-detail-redirect/report-of-the-who-china-jointmission-oncoronavirus-disease-2019-(covid-19).

3. Onder G, Rezza G, Brusaferro S (2020) Case-Fatality Rate and Characteristics of Patients Dying in Relation to COVID-19 in Italy. JAMA 323: 1775-1776.

4. Zhou F, Yu T, Du R (2020) Clinical course and risk factors for mortality of adult inpatients with COVID-19 in Wuhan, China: a retrospective cohort study. Lancet 395: 1054-1062.

5. Guan WJ, Liang WH, Zhao Y, Heng-Rui Liang, Zi-Sheng Chen, et al. (2020) Comorbidity and its impact on 1590 patients with Covid-19 in China: A Nationwide Analysis. Eur Respir J 55: 2000547.

6. Aldridge RW, Lewer D, Katikireddi SV, Rohini Mathur, Neha Pathak, et al. (2020) Black, asian and minority ethnic groups in England are at increased risk of death from COVID-19: indirect standardisation of NHS mortality data. Wellcome Open Res 5: 88.

7. Chinnadurai R, Ogedengbe O, Agarwal P (2020) Older age and frailty are the chief predictors of mortality in COVID-19 patients admitted to an acute medical unit in a secondary care setting- a cohort study. BMC Geriatr 20: 409.

8. Ayoubkhani D, Khunti K, Nafilyan V (2021) Epidemiology of post-COVID syndrome following hospitalisation with coronavirus: a retrospective cohort study. https://doi.org/10.1 101/2021.01.15.21249885.

9. Donnelly J, Qing Wang X, Iwashyna T (2020) Readmission and Death After Initial Hospital Discharge Among Patients with COVID-19 in a Large Multihospital System. JAMA 2020: e2021465.

10. UK Government. Coronavirus (COVID-19) in the UK: daily update. https://coronavirus.data.gov.uk.
Copyright: (02021 Ghosh S, et al. This is an open-access article distributed under the terms of the Creative Commons Attribution License, which permits unrestricted use, distribution, and reproduction in any medium, provided the original author and source are credited. 